\title{
Healthy mind in healthy body? A review of sensorimotor -cognitive interdependencies in old age
}

\author{
Sabine Schäfer • Oliver Huxhold • Ulman Lindenberger
}

Received: 24 April 2006 / Accepted: 28 June 2006/ Published online: 12 August 2006

(C) EGREPA 2006

\begin{abstract}
We review four broad lines of research on couplings between sensorimotor and cognitive aging, with an emphasis on methodological concerns. First, correlational cross-sectional and longitudinal data indicate increasing associations between sensorimotor and cognitive aspects of behavior with advancing age. Second, older adults show greater performance decrements than young adults when sensorimotor and cognitive tasks or task components need to be performed concurrently rather than in isolation. Third, aerobic fitness interventions produce positive transfer effects on cognition that are particularly pronounced for tasks with high demands on attention and executive control. Fourth, neuroscience findings from animal models and humans have identified aging-sensitive structural and functional circuitries that support cognitive functions and are enhanced by higher levels of sensorimotor functioning. We conclude that sensorimotor and cognitive aging are causally related and functionally interdependent and that age-associated increments in cognitive resource demands of sensorimotor functioning are malleable by experience.
\end{abstract}

Keywords Cognitive aging · Motor aging · Brain aging · Aerobic fitness $\cdot$ Sensorimotor-cognitive couplings

\footnotetext{
S. Schäfer $\cdot$ O. Huxhold $\cdot$ U. Lindenberger

Center for Lifespan Psychology,

Max Planck Institute for Human Development,

Berlin, Germany

S. Schäfer $(\bowtie)$

Max Planck Institute for Human Development,

Lentzealle 94,

14195 Berlin, Germany

e-mail: Schaefer@mpib-berlin.mpg.de
}

Normal aging is associated with losses in the functional integrity of sensory, sensorimotor, and cognitive domains. Each of these losses has been studied extensively at behavioral and physiological levels of analysis. However, considerably less attention has been paid to the question whether senescent changes are causally shared and functionally coupled across domains [53]. In this review, we present causal and functional couplings between sensorimotor and cognitive aging. We report evidence from correlational studies, dual-task experiments, aerobic fitness interventions, and select neuroscience work in animals and humans, and we also discuss methodological issues that should be considered when conducting this kind of research. Findings from all of these disparate lines of research converge on the notion that sensorimotor and cognitive aging form an intricate link and are malleable by experience.

\section{Adult age differences in correlations among sensorimotor, sensory, and cognitive performance}

Aging is associated with decrements in cognitive and sensorimotor functioning [51, 68]. In addition, several correlational studies have reported an increase in the association between sensory, sensorimotor, and cognitive functions from early adulthood to old age $[2,3,9,53]$. On the sensory and sensorimotor side, measures included visual acuity, auditory acuity, grip strength, strength in the lower limbs, and forced expiratory volume; one study also included the tactile modality [48]. Using hierarchical linear regression, Baltes and Lindenberger [9] assessed the proportion of shared variance between sensory functioning, operationalized as visual and auditory acuity, and intelli- 
gence, which was assessed by 14 tests indicating perceptual speed, reasoning, episodic memory, verbal knowledge, and verbal fluency. On average, the proportion of interindividual differences in intelligence associated with sensory functioning increased from $11 \%$ in 25 - to 69 -year-olds to $31 \%$ in older ages (70-103 years). Moreover, in the sample of older adults, sensory functioning was a stronger predictor of psychometric intelligence than a comprehensive set of sociobiographical factors.

Based on these correlational associations and informed by theoretical conceptions about biological aging, Anstey and Smith [2] and Anstey et al. [3] have hypothesized that sensory and sensorimotor declines may precede and predict cognitive decline. Similarly, Lindenberger and Baltes [53] have proposed that some of the brain mechanisms causing senescent changes are shared among sensory, sensorimotor, and cognitive functions, but have refrained from assigning temporal or causal priority to any of the three domains ("common cause hypothesis"). If mechanisms of biological aging compromise the functional integrity of the brain in some general way or affect specific structures or processes of the brain that are implicated in sensory, sensorimotor, and cognitive functions, then these functions will show various degrees of senescent covariation, depending upon the relative importance of shared causality. One particular mechanism proposed in this context are neurochemical changes leading to lower signal-to-noise ratios, less distinct representations, and a generalized dedifferentiation of functional brain states [47, 49].

However, in interpreting these findings, the inferential limitations of cross-sectional data sets need to be taken into account. As has been recognized for quite some time [31, $32,38,71]$, the prominence of developmental mechanisms common to different domains or the dimensionality of developmental change cannot be ascertained by crosssectional correlational methods $[31,32,34,37,39,44$, $54,77]$. The degree to which two variables are correlated in an age-heterogeneous data set is strongly influenced by the similarity of their mean age trends and the association between the two variables that is orthogonal to age $[54,77]$. In connection to this, the degree to which individual differences in one variable predict age-related variance in another is a quadratic function of the partial correlation between the two variables controlling for age [54].

Longitudinal panel data circumvent some, but not all, of the shortcomings of cross-sectional data. If loss in function is driven by causes shared across domains, interindividual differences in change ought to be correlated across domains. This prediction has received partial support by multivariate longitudinal structural equation modeling analyses that found moderate to strong correlations among changes in sensory and cognitive functioning [4, 5, 28]. For example, Ghisletta and Lindenberger [28] found that age changes in two sensory abilities, close vision and distance vision, and two intellectual abilities, perceptual speed and verbal knowledge, were correlated across a period of up to 6 years in a sample of old and very old adults. Moreover, the analysis of dynamic change components using nonlinear equations provided tentative evidence that neither domain was dominating change in the other because leadlag relations went in both directions [28].

When evidence based on cross-sectional and traditional longitudinal panel research designs is brought to bear upon questions regarding within-person developmental dynamics and dimensionality, the assumption is made that betweenperson structures generalize to within-person structures [55, 57]. For example, the notion of common causation in the context of sensory, sensorimotor, and cognitive aging primarily refers to domain-general mechanisms operating across time within aging individuals, rather than to forces operating between individuals that are subject to senescent changes. Generalizing from between-person to withinperson variation is legitimate only when certain formal conditions, often summarized under the heading of ergodicity, are met [34, 54, 55, 64, 65]. Testing this assumption empirically requires dramatic shifts in research strategy and statistical methodology that change the empirical base of developmental analyses from interindividual differences to intraindividual variations [50, 54, 55, 57, 58, 62, 64]. First, single individuals have to be assessed repeatedly and intensively on measures of interest so that within- and across-domain associations and temporal dynamics can be estimated within single persons. The similarity and age dependence of within-person structures and their match to between-person structure can then be gauged in a second step. First steps in this research direction have been undertaken. With respect to the link between sensorimotor and cognitive performance, $\mathrm{Li}$ et al. [50] demonstrated in a sample of older adults (mean $=75.71$ years, $\mathrm{SD}=6.93$ years) that the magnitude of intraindividual biweekly variations in walking performances and episodic memory was at least half as great as the interindividual variation found across persons. The level of intraindividual variation in walking performance was higher among older persons in the sample than among the younger ones. Moreover, the level of intraindividual biweekly fluctuations in walking steps predicted uniquely interindividual differences in text recall above the prediction of the average walking performance. These results speak for the importance of treating intraindividual response variations as important empirical variables that enhance an understanding of cognitive and sensorimotor aging.

In another recently completed study [52], intraindividual response variations in postural control and working memory of 18 young adults and 18 older adults were assessed across 45 days. Preliminary results showed that the 
cross-domain couplings of sensorimotor and cognitive fluctuations observed within individuals across time differed strongly between persons. Cross-domain intraindividual couplings tended to be stronger for individuals of lower postural control performance than for high-performing individuals [35].

\section{Experimental evidence on resource competition between sensorimotor and cognitive aspects of behavior}

Dual-task methodology is often used to examine whether the execution of different tasks critically depends on a common set of mechanisms acting as a limited resource [79]. If sensorimotor aspects of behavior require greater investments of cognitive resources with advancing adult age, older adults should have greater difficulties than young adults when sensorimotor and cognitive tasks or task components need to be performed conjointly rather than separately. Given that elementary and coordinate aspects of sensory and sensorimotor performance deteriorate with advancing age, it has been argued that older adults need to invest increasing amounts of cognitive resources into sensorimotor functioning. Lindenberger et al. [56] denoted this tendency as the aging-induced permeation of sensorimotor functioning with cognition (see also early remarks by Alan Welford [78], pp. 186-187). For instance, when crossing a busy street, an average 85-year-old adult will need to invest more cognitive resources into sensory and sensorimotor aspects of behavior than an average 20-year-old adult. Hence, the older adults may be forced to interrupt a conversation with a friend and resume it only after the street has been crossed.

Lindenberger et al. [56] and Li et al. [46] listed a number of criteria that should be met to assess adult age differences in functional interdependencies between sensorimotor and cognitive domains of functioning with high internal and external validity. To increase the internal validity of claims regarding age differences in dual-task costs (DTCs), it is advisable to administer single and dual tasks at overlapping levels of difficulty [40] or to equate young and old participants on single-task performance by means of adaptive testing procedures or differential task exposure [38]. In addition, it is generally more appropriate to express DTCs in relative, rather than absolute, terms (e.g., as percentage of loss relative to single-task performance; $\mathrm{cf}$. [8]). To enhance generalizability (e.g., external validity), the tasks used in the laboratory should mimic the kind of challenges encountered in everyday multitasking situations. Also, research participants should be explicitly instructed to focus more strongly on one task than on the other to test the extent to which resource allocation variations are amenable to conscious control or automatically triggered by task affordances. Many of the studies reported below conform to most of these criteria.

Lindenberger et al. [56] instructed groups of young (2030 years), middle-aged ( $40-50$ years), and old (60-70 years) adults in an imagery-based mnemonic technique for the serial recall of word lists, and then trained skilled memory performance with this technique until participants in all age groups approximated or attained a prespecified criterion performance. Individuals were also asked to walk as quickly and accurately as possible on two narrow tracks of different path complexity. Training of both component tasks under single-task conditions assured that participants were familiar with the tasks before initiation of the dualtask phase. Age-based increments in DTCs were already noticeable among middle-aged adult relative to young adults, and further increased from middle-aged adults to old age, both with respect to walking and with respect to memory performance (see Fig. 1). Apparently, the aginginduced de-automatization of sensorimotor functioning and the concurrent increase in cognitive control demands is not confined to the age periods of old and very old but can be observed throughout the adult life span, if the difficulty of the sensorimotor task is sufficiently high.

$\mathrm{Li}$ et al. [45] replicated and extended these findings in 20- to 30- and 60- to 75-year-old adults, again combining skilled memory performance with walking on a narrow oval track. At times, task complexity was adjusted to each individual's single-task performance level by varying presentation times for memory items (memory task) or by placing obstacles on the track (walking task). Performance in both age groups deteriorated when the two tasks had to be performed concurrently under difficult task conditions. Age differences in DTCs were more pronounced in the memory task than in the walking task, suggesting that older adults prioritized the sensorimotor domain, possibly to protect themselves from falls. This performance pattern was interpreted as an adaptive application of resources by the older age group, since falls can lead to severe consequences in the elderly [73].

Brown et al. [15] compared young and older adults' ability to stabilize their posture in four conditions of postural threat while concurrently performing a spatial working memory task. In addition, the authors measured galvanic skin conductance to indicate changes in physiological arousal. An index of task prioritization was calculated, based on relative changes in cognitive and balance performance across testing conditions. In agreement with $\mathrm{Li}$ et al. [45], the authors found that older adults were more likely to prioritize postural control over secondary task performance under conditions of increased postural threat than younger adults were. Related findings by Krampe et al. [43] suggest that the ability to adaptively 


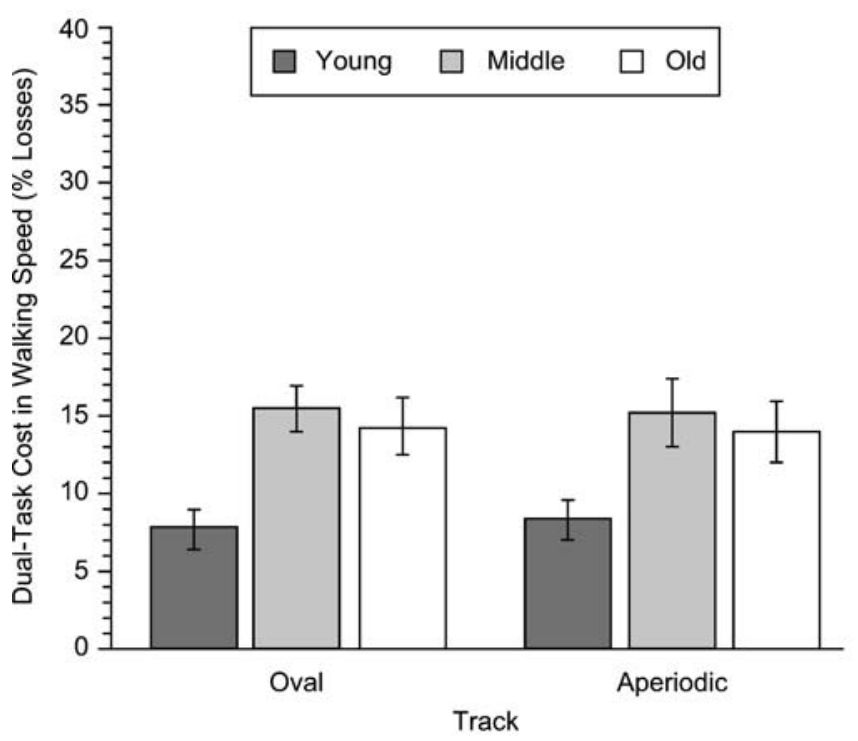

Fig. 1 Age differences in dual-task costs during cognitive and sensorimotor performance. The left panel depicts dual-task costs in walking speed, and the right panel depicts dual-task costs in memory performance. For the walking task, participants either walked on an

allocate resources to sensorimotor vs cognitive aspects of behavior is preserved to some degree at least in adults suffering from Alzheimer's disease with a dysexecutive syndrome. When asked to perform a working memory task while keeping an upright posture on a moving balance platform, dementia patients and older adults showed little or no reductions in their postural stability while their memory performance decreased significantly.

In another dual-task study, Brauer et al. [13] compared balance-impaired and healthy older adults. The balance task consisted of stabilizing oneself after mild balance disturbances caused by sudden movements of a platform. The secondary task required that participants reacted verbally to auditory stimuli. As revealed by delayed reactions to the auditory stimuli, balance recovery required more attention in the balance-impaired than in the healthy group. In addition, while balance performance remained unaltered among the healthy adults, the balanceimpaired older adults took longer to regain balance under dual-task than under single-task conditions. The authors argue that concurrent attentional demands may result in postural instability and contribute to falls among balanceimpaired elderly individuals [60].

However, performance does not always decrease when cognitive and sensorimotor tasks are performed concurrently. Huxhold et al. [36] could show that young and older adults swayed less when they were concurrently performing an easy cognitive task relative to a single-task baseline, where the focus of attention was explicitly directed toward the postural control task itself. When the cognitive task was

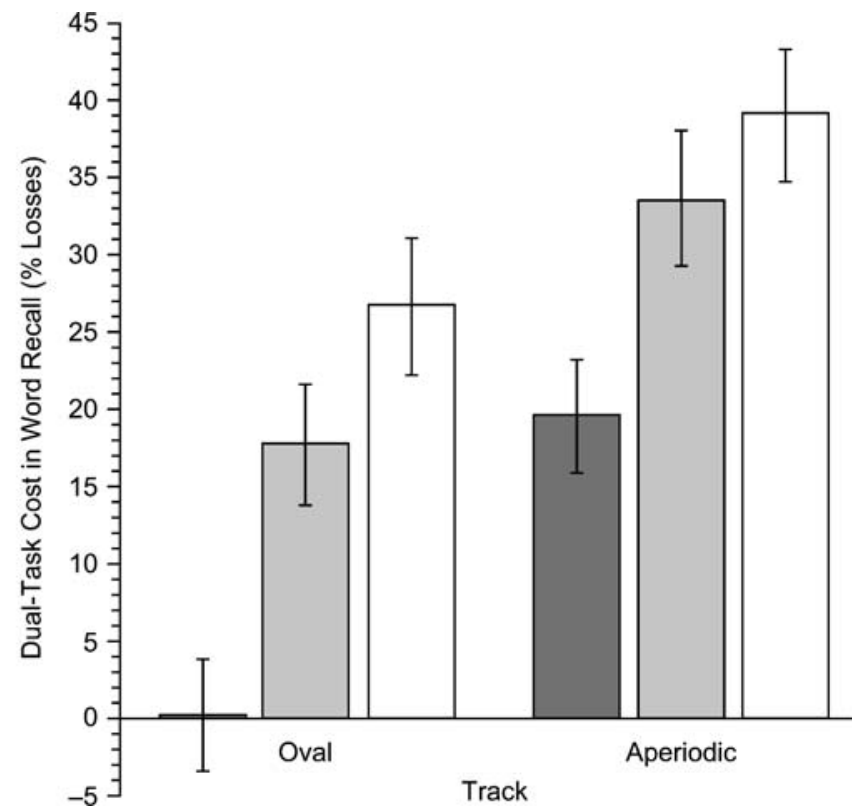

oval track (low task complexity) or on an aperiodic track (high task complexity). Error bars depict standard errors of the mean. Modified after [56]

more demanding, older adults showed an increase in their body sway, but young adults did not. The authors note that mild forms of dual-tasking may be the rule for many manifestations of sensorimotor functions in everyday life. Therefore, performing a concurrent cognitive task that is relatively low in cognitive demand may benefit postural control by directing individuals' overt attention away from postural control processes that are usually carried out automatically. However, further increments in the cognitive demands of the secondary task will eventually result in attentional resource competition, thereby hampering the regulation of postural sway.

Lövdén et al. [59] asked younger and older men to walk on a treadmill and learn their way through a virtual mazelike museum that was projected in front of them. Participants were either walking with or without support (holding on to a handrail). Navigation load increased trunkangle variability for older adults only, indicating that their gait was less stable when concurrently navigating in the maze instead of only walking on the treadmill. At the same time, age differences in navigational place learning were more pronounced when participants were walking without support (see Fig. 2). Specifically, handrail support had no reliable effect on way-finding performance among young adults but improved older adults' performance, when measured in distance walked to reach the learning criterion, by about $25 \%$.

In sum, age-comparative studies suggest that interdependencies between sensorimotor and cognitive tasks or task components increase from early to late adulthood. 


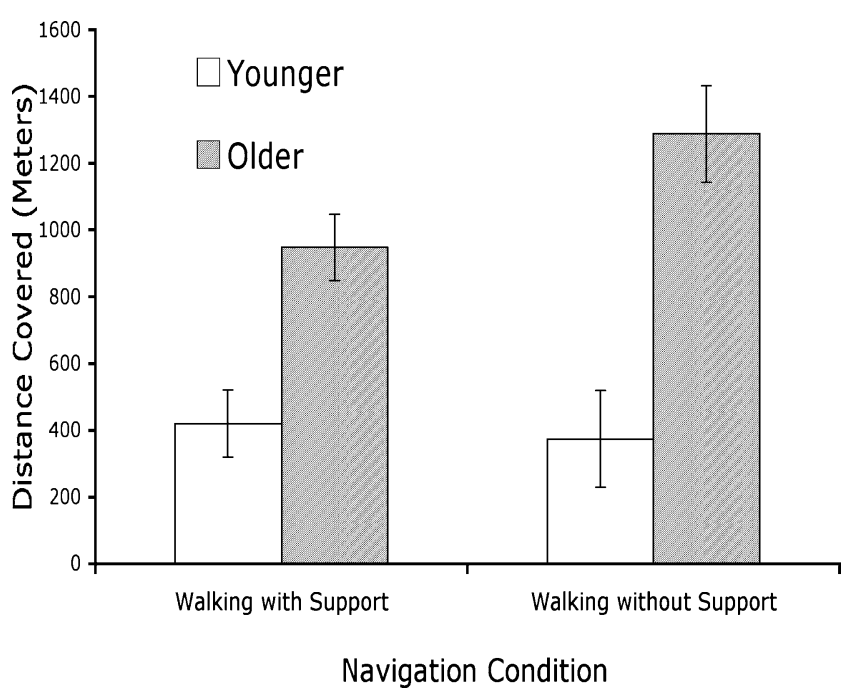

Fig. 2 Age differences in navigation performance vary by walking support. The two bars to the left depict navigation performance (distance walked until criterion is reached) when participants were allowed to use a handrail, while the other two bars to the right depict navigation performance participants were walking without support. Error bars depict standard errors of the mean. Modified after [59]

Apparently, sensorimotor aspects of behavior are more attention-demanding in older adults than in young adults, and attentional resources are reduced. As suggested by the cognitive permeation hypothesis of sensorimotor performance, these two tendencies lead to increased competition between sensorimotor and cognitive tasks or task components for scarce attentional resources. To some extent, older adults appear to cope with increasing and ubiquitous resource competition by deliberately shifting attention to either cognitive or sensorimotor aspects of behavior, depending upon task emphasis. However, deliberate resource allocation may be overruled by mandatory investments of attentional resources into sensorimotor aspects of behavior when physical integrity is at stake [60].

\section{From body to mind? Effects of aerobic fitness on cognition}

Correlational and experimental studies reviewed so far point to an increasingly close connection between sensorimotor and cognitive domains of functioning with advancing adult age. This age-associated trend raises the question whether intervention-related improvements in one of these two domains are accompanied by improvements in the other. For at least two reasons, such positive acrossdomain transfer effects seem theoretically plausible. First, increasing efficiency of processes in either domain should reduce competition for attentional resources. That is, if sensorimotor functioning demands less attention through sensorimotor intervention or if attentional processes become more efficient through cognitive intervention, then these changes should reduce resource competition, with potentially beneficial effects on performance in the other domain. Second, to the extent that sensorimotor and cognitive aspects of behavior partly influence and depend on a common substrate such as the prefrontal cortices of the brain, interventions in either of the two domains may come to light as more or less viable access routes to ameliorate the functional status of the substrate, which in turn may positively affect functioning in both domains.

Empirically, the route from sensorimotor interventions to cognitive effects, especially if targeted at aerobic fitness, appears to be more promising than the route from cognitive interventions to sensorimotor effects. Several cross-sectional studies with older adults suggest a link between physical fitness and cognitive performance [10, 11, 17, 23, 33]. For instance, studies by Spirduso [74] and by Spirduso and Clifford [75] showed that older racquet sportsmen or older runners were significantly faster on simple choice and movement response times than older nonexercisers. Furthermore, in a prospective longitudinal study with 30 volunteers approaching age 65 , Rogers et al. [72] found that individuals who participated in regular physical activities sustained more constant levels of cerebral blood flow and higher cognitive performance scores than those who did not participate in regular physical activities.

More recently, planned interventions have been carried out to directly examine the effects of changes in aerobic fitness on cognitive performance among older adults. Several reviews that summarize this line of research are available $[18,20,25,26,29,30,62]$. Colcombe and Kramer [18] performed a meta-analysis including 18 intervention studies published between 1966 and 2001. Overall, aerobic fitness training was found to have robust but selective benefits for cognition. The magnitude of these effects was moderated by factors like the type of intervention, duration of the fitness training intervention, duration of training sessions, and sex. Specifically, the beneficial effects of fitness training were found to be most pronounced for executive processes, followed by spatial and speeded tasks. Also, individuals who participated in combined strength and aerobic training regimes improved their cognitive performance reliably more than individuals who only trained one fitness regime. Relatively brief training programs with an average of 2 months provided about as much of an effect on cognitive performance as moderate training, but not quite as much as long-term training programs that lasted more than 6 months. Programs with sessions lasting for more than 30 min of training were more effective than programs with shorter sessions. Finally, effects of fitness training on cognitive performance were greater with samples that contained more women than men 
and when participants belonged to the mid-old age category (i.e., 66-70 years).

Interestingly, a recent meta-regression analysis reported by Etnier et al. [26] failed to support the hypothesis that improvements in aerobic fitness contribute to improvements in cognitive performance. The authors investigated the relationship between fitness effect sizes and cognitive effect sizes across different studies. No significant linear or curvilinear relationship between fitness effect sizes and cognitive effect sizes was found for studies using cross-sectional designs or posttest comparisons. In addition, across intervention studies, the authors found a significant negative relation between aerobic fitness and cognitive performance effect sizes for pre-post comparisons. In other words, intervention studies showing relatively large improvements in cardiovascular fitness showed rather less pronounced increases in cognitive performance and vice versa. The authors conclude that future research should investigate the dose-response relationship between aerobic fitness and cognitive performance in greater detail.

We fully agree that the functional relation between changes in fitness and changes in cognition is far from being fully understood and needs further study. At the same time, it needs to be noted that the results of meta-regression analyses of the kind reported by Etnier et al. [26] are open to a great variety of possible interpretations. When regressing different effect sizes across different studies onto another, the outcome will partly depend on the ratio of between-person differences in cognitive performance and between-person differences in aerobic fitness within individual studies. From a multilevel modeling perspective, the results represent level 2 effects and may not be congruent with level 1 effects (e.g., did those individuals within a study who improved more in fitness also improve more in cognition than other individuals within the same study who improved less in fitness?). For instance, the various studies used different cognitive tasks and different fitness measures, and those studies that used more change-sensitive fitness measures may have used less change-sensitive cognitive measures, perhaps reflecting the differences in the primary field of expertise of different research teams. Thus, the interpretability of meta-regression analyses is restricted by differences in task design, measurement reliability, and sample characteristics. To obtain a better understanding of the closeness and functional form of the fitness-cognition link, the fine-grained investigation of the dose-response relationships between aerobic fitness and cognitive performance envisioned by Etnier et al. [26] is best undertaken at the within-person level.

Generally, aerobic fitness intervention studies are conducted with cognitively healthy older adults who do not engage in regular physical activity before the intervention
$[41,42,61,66]$. These individuals are then assigned randomly to an exercise or a control group. If the cognitive performance of the exercise group improves more strongly than the performance of the control group, this improvement is likely to be caused by exercise itself or by factors associated with exercise. For instance, Kramer et al. [42] randomly assigned 124 previously sedentary adults aged 60 to 75 years either to an aerobic (walking) or an anaerobic (stretching) exercise group. In either case, training lasted over a period of 6 months. Cognitive tasks aimed at assessing executive control processes such as planning, scheduling, inhibiting, maintaining, and coordinating information were administered before and after the intervention. At posttest, cardiovascular fitness, as indexed by the maximum rate of oxygen consumption, had improved in the walking group but not in the stretching group. In the cognitive domain, performance on task conditions that assess executive control processes improved significantly among individuals in the aerobic exercise group, but did not improve among individuals in the stretching group. At the same time, performance on task conditions that impose lower demands on executive control such as simple reaction time did not differ between the two exercise groups at posttest. This apparent specificity of the aerobic training may help to explain why Madden et al. [61], who used tests of attention and episodic memory, failed to find improvements in cognitive performance as a function of aerobic exercise intervention.

Moul et al. [66] assigned 30 sedentary older adults to either a walking group, a weight training group, or a placebo control group and asked the two exercise groups to train for 30-60 min, 5 days/week over a period of 16 weeks. At baseline and after the 16 weeks of training, individuals in all groups were tested with the Ross Information Processing Assessment test, which consists of ten subtests measuring memory, temporal and spatial orientation, and problem solving skills. Individuals in the walking group showed significant improvements in maximum oxygen consumption, whereas individuals in the other groups did not. In the exercise group, cognitive improvements were found as well, whereas both the weight training and the control groups showed little change in cognitive tests scores. Follow-up analyses revealed that the cognitive improvements in the walking group were primarily due to significant improvements in the two most attentiondemanding dimensions of the test.

Hawkins et al. [30] asked younger and older adults to perform time-sharing and attentional flexibility tasks. In one study, individuals who participated in a 10-week aquatic exercise program showed substantially greater improvements in alternation speed and time-sharing efficiency than same-aged participants in the control group. Again, the two groups did not differ in the amount of 
improvements in single-task performance, suggesting that the effect of exercise was specific to dual-task processing.

What are the mechanisms that mediate the beneficial effects of aerobic fitness interventions on cognitive performance? Why is it the case that these effects appear to be especially pronounced for tasks that assess the efficiency of executive control? Several lines of research point to relevant brain areas and mechanisms but their relative importance has not yet been fully understood. With respect to human evidence, a recent cross-sectional study by Colcombe et al. [19] examined older adults' brain tissue density as a function of age and aerobic fitness. Robust declines in tissue densities with age were found in the frontal, parietal, and temporal cortices. These losses were substantially reduced in individuals with greater aerobic fitness, even after controlling for potentially confounding variables such as years of education. The attenuating effects of aerobic fitness on age-based reductions in tissue density were most pronounced for brain areas that showed the strongest negative association between tissue density and age. In other words, the brain areas that were highly susceptible to normal aging also turned out to be more modifiable by fitness-related factors.

In sum, aerobic fitness appears to have beneficial structural and functional consequences on the human cortex and the prefrontal cortices, in particular, demonstrating the continued existence of neural plasticity in adulthood and old age. In support of this line of reasoning, it has been found that improvements on cognitive task performance observed after aerobic fitness interventions were accompanied by concomitant changes in patterns of cortical activation while performing these tasks. Apparently, activation patterns of older adults are more similar to those of young adults after the intervention [21]. Moreover, in a study with young adults, learning to juggle led to transient increases in gray matter in brain regions that presumably code visual motion information [22]. Taken together, this evidence underscores the continued existence of cortical plasticity in adulthood and old age.

Animal models with rodents have examined changes in cognitive performance and brain functioning as a function of enriched environments and aerobic exercise. Increased aerobic fitness in these animals is associated with increases in capillary density in the cerebellum [12], enhanced cortical high-affinity choline uptake and increased dopamine receptor density [27], increased brain-derived neurotrophin factor gene expression [1, 67], and with increases in the number of new cells in the hippocampus $[16,76]$. It seems plausible that some of the cellular, molecular, and neurochemical mechanisms observed in rodents also operate in humans and contribute to the sensorimotor, perceptual, and cognitive changes in response to aerobic fitness interventions. In fact, such mechanisms may act as common cause [53] that affects performance across different domains of functioning. At the same time, the relative importance of different mechanisms, such as angiogenesis and synaptogenesis, remains to be explored. High-field structural and functional magnetic resonance tomography, including magnetic resonance spectroscopy to measure metabolites of synaptogenesis such as $N$-acetylaspartate, $N$-acetylaspartylglutamate, glutamate, and taurine, may help to clarify these issues.

\section{Mind and body in old age: integration and future research directions}

This review has discussed the connection between sensorimotor and cognitive functions in old age. Based on the available evidence, we conclude that normal aging strengthens the link between the two functional domains through two separable but closely connected and interacting developmental processes.

First, there can be no doubt that the functional link between sensorimotor and cognitive domains increases with advancing adult age. As basic and coordinative sensory and sensorimotor mechanisms become increasingly less efficient and more error-prone with advancing age, sensorimotor skills with a considerable degree of automaticity, such as the crossing of a busy street, increasingly de-automatize and disassemble so that their execution imposes increasingly high demands on mechanisms of cognitive control such as attentional and executive processes. At the same time, mechanisms of cognitive control also undergo marked deterioration with advancing age. Thus, behavioral aging is marked by the quandary of increasing demands on decreasing resources [56]. Note that the dynamics of this developmental process would lead to increasingly close connections between sensorimotor and cognitive aspects of behavior even if brain changes associated with functional losses in these domains were completely separate.

Second, the etiological link between sensorimotor and cognitive domains of functioning is also increasing with advancing adult age. Evidently, the anatomical, chemical, and functional changes of the aging brain often transcend the terminological and conceptual boundaries that separate sensory, motor, perceptual, and cognitive aspects of behavior. Older brains show signs of neural decline, like cerebral atrophy in the frontal cortex, gray matter loss [70], losses in structural connectivity, decreases in dopamine receptor density [6, 7], and demyelination ([69]; for a summary, see [68]). In addition, changes in functional connectivity can be observed. To the extent that these changes affect more than one domain of functioning, they limit performance across domains, and qualify as a common cause [53]. 
In this situation, the benefits of aerobic exercise may affect both functional and etiological connections between body and mind. From a functional perspective, aerobic exercise may reduce the attentional demands of sensorimotor functioning by strengthening the limbs and aiding coordination and postural control mechanisms [63], thereby alleviating the attentional mortgage of the body on the mind. From an etiological perspective, aerobic exercise may provoke anatomic, chemical, and functional alterations in the brain that counteract senescent changes in the brain, and thereby enhance aspects of cognitive performance that show pronounced losses with age, such as attentional control. Interestingly, cognitive interventions generally have resulted in less consistent and less generalizable performance gains on cognition than fitness interventions. Moreover, the potential effect of cognitive interventions on various aspects of sensorimotor performance, such as postural control under dual-task conditions, has not been investigated so far.

Future studies need to shed further light on functional and etiological links between sensorimotor and cognitive aging and their interaction. First, aerobic fitness interventions should assess cognitive performance before and after fitness training with and without the concurrent performance of an attentionally challenging sensorimotor task such as walking on a narrow track or balancing on an ankle-disc board. If aerobic fitness interventions reduce the attentional demands of sensorimotor tasks, in addition to cognition-enhancing effects on the brain, then intervention effects should be more pronounced for cognitive tasks performed during sensorimotor challenge. Second, intervention effects may be further enhanced by combining aerobic training with cognitive tasks that activate, in part, the same brain structures that have been found to be altered by aerobic exercise. In other words, and as suggested by ingenious animal models [14, 24], combinations of sensorimotor and cognitive intervention may prove to be more effective than either intervention in itself. Third, the diminishing returns of aerobic fitness on cognitive performance deserve greater attention. It needs to be ascertained more precisely whether fitness gains become inconsequential for cognition if fitness exceeds a certain threshold. Taken together, these lines of research will help us to further understand the intimate and intricate links between a healthy body and a healthy mind.

\section{References}

1. Adlard PA, Perreau VM, Cotman CW (2005) The exerciseinduced expression of BDNF within the hippocampus varies across the life-span. Neurobiol Aging 26:511-520
2. Anstey KJ, Smith GA (1999) Interrelationships among biological markers of aging, health, activity, acculturation, and cognitive performance in late adulthood. Psychol Aging 14:605-618

3. Anstey KJ, Lord SR, Williams P (1997) Strength in the lower limbs, visual contrast sensitivity, and simple reaction time predict cognition in older women. Psychol Aging 12:137-144

4. Anstey KJ, Hofer SM, Luszcz MA (2003) A latent growth curve analysis of late-life sensory and cognitive function over 8 years: evidence for specific and common factors underlying change. Psychol Aging 18:714-726

5. Anstey KJ, Hofer SM, Luszcz MA (2003) Cross-sectional and longitudinal patterns of dedifferentiation in late-life cognitive and sensory function: the effects of age, ability, attrition, and occasion of measurement. J Exp Psychol Gen 132:470-487

6. Bäckman L, Farde L (2005) The role of dopamine systems in cognitive aging. In: Cabeza R, Nyberg L, Park DC (eds) Cognitive neuroscience of aging: linking cognitive and cerebral aging. Oxford University Press, New York, pp 58-84

7. Bäckman L, Ginovart N, Dixon RA et al (2000) Age-related cognitive deficits mediated by changes in the striatal dopamine system. Am J Psychiatryyiat 157:635-637

8. Baddeley A, Della Sala S, Gray C, Papagno C, Spinnler H (1997) Testing central executive functioning with a pencil-and-paper test. In: Rabbitt PMA (ed) Methodology of frontal and executive functions. Psychology Press, Hove, England, pp 61-80

9. Baltes PB, Lindenberger U (1997) Emergence of a powerful connection between sensory and cognitive functions across the adult life span: a new window to the study of cognitive aging? Psychol Aging 12:12-21

10. Bashore T (1989) Age, physical fitness and mental processing speed. In: Lawton MP (ed) Annual review of gerontology and geriatrics. Springer, Berlin Heidelberg New York, pp 120-144

11. Baylor A, Spirduso WW (1988) Systematic aerobic exercise and components of reaction-time in older women. J Gerontol 43:121-126

12. Black JE, Isaacs KR, Anderson BJ, Alcantara AA, Greenough WT (1990) Learning causes synaptogenesis, whereas motor activity causes angiogenesis in cerebellar cortex of adult rats. Proc Natl Acad Sci U S A 87:5568-5572

13. Brauer SG, Woollacott M, Shumway-Cook A (2001) The interacting effects of cognitive demand and recovery of postural stability in balance-impaired elderly persons. J Gerontol A Biol Sci Med Sci 56(8):M486-M496

14. Brown J, Cooper-Kuhn CM, Kempermann G et al (2003) Enriched environment and physical activity stimulate hippocampal but not olfactory bulb neurogenesis. Eur J Neurosci 17:2042-2046

15. Brown LA, Sleik RJ, Polych MA, Gage WH (2002) Is the prioritization of postural control altered in conditions of postural threat in younger and older adults? J Gerontol A Biol Sci Med Sci 57A:M785-M792

16. Churchill JD, Galvez R, Colcombe S, Swain RA, Kramer AF, Greenough WT (2002) Exercise, experience and the aging brain. Neurobiol Aging 23:941-955

17. Clarkson P (1978) The effect of age and activity level on simple and choice fractional reaction time. Eur J Appl Physiol 40:17-25

18. Colcombe S, Kramer AF (2003) Fitness effects on the cognitive function of older adults: a meta-analytic study. Psychol Sci 14:125-130

19. Colcombe S, Erickson KI, Raz N et al (2003) Aerobic fitness reduces brain tissue loss in aging humans. J Gerontol A Biol Sci Med Sci 58A:176-180

20. Colcombe S, Kramer AF, McAuley E, Erickson KI, Scalf P (2004) Neurocognitive aging and cardiovascular fitness: recent findings and future directions. J Mol Neurosci 24:9-14 
21. Colcombe SJ, Kramer AF, Erickson KI et al (2004) Cardiovascular fitness, cortical plasticity, and aging. Proc Natl Acad Sci U S A 101:3316-3321

22. Draganski B, Gaser C, Busch V, Schuierer G, Bogdahn U, May A (2004) Changes in grey matter induced by training. Nature 427:311-312

23. Dustman R, Emmerson R, Shearer D (1994) Physical activity, age, and cognitive neurophysiological function. J Aging Phys Act 2:143-181

24. Ehninger D, Kempermann G (2003) Regional effects of wheel running and environmental enrichment on cell genesis and microglia proliferation in the adult murine neocortex. Cereb Cortex 13:845-851

25. Etnier JR, Salazar W, Landers DM, Petruzzello SJ, Han M, Nowell P (1997) The influence of physical fitness and exercise upon cognitive functioning: a meta-analysis. J Sport Exerc Psychol 19:249-277

26. Etnier JL, Nowell PM, Landers DM, Sibley BA (2006) A metaregression to examine the relationship between aerobic fitness and cognitive performance. Brain Res Brain Res Rev 52(1):119-130. DOI 10.1016/j.brainresrev.2006.01.002

27. Fordyce DE, Farrar RP (1991) Physical activity effects on hippocampal and parietal cholinergic function and spatial learning in F344 rats. Behav Brain Res 43:115-123

28. Ghisletta P, Lindenberger U (2005) Exploring structural dynamics within and between sensory and intellectual functioning in old and very old age: longitudinal evidence from the Berlin Aging Study. Intelligence 33:555-587

29. Hall CD, Smith AL, Keele SW (2001) The impact of aerobic activity on cognitive function in older adults: a new synthesis based on the concept of executive control. Eur J Cogn Psychol 13:279-300

30. Hawkins HL, Kramer AF, Capaldi D (1992) Aging, exercise, and attention. Psychol Aging 7:643-653

31. Hertzog C (1985) An individual differences perspective: implications for cognitive aging research in gerontology. Res Aging 7:745

32. Hertzog C (1996) Research design in studies of aging and cognition. In: Birren JE, Schaie KW, Abeles RP, Gatz M, Salthouse TA (eds) Handbook of the psychology of aging. Academic, San Diego, CA, pp 24-37

33. Hillman CH, Weiss EP, Hagberg JM, Hatfield BD (2002) The relationship of age and cardiovascular fitness to cognitive and motor processes. Psychophysiology 39:303-312

34. Hofer S, Sliwinski M (2001) Understanding aging: an evaluation of research designs for assessing the interdependence of agerelated changes. Gerontology 47:341-352

35. Huxhold O, Li S-C, Lindenberger U (2005) Postural control fluctuations across days: within-person analyses of the effects of age and fluctuations in cognition. 58th Annual Scientific Meeting of The Gerontological Society of America. Orlando, Florida

36. Huxhold O, Li S-C, Schmiedek F, Lindenberger U (2006) Dualtasking postural control: aging and the effects of cognitive demand in conjunction with focus of attention. Brain Res Bull 69:294-305

37. Kalveram KT (1965) Alterations in factorial structure through "simultaneous interference." Arch Gesamte Psychol 117:296-305

38. Kliegl R, Lindenberger U (1993) Modeling intrusions and correct recall in episodic memory: adult age differences in encoding of list context. J Exp Psychol Learn Mem Cogn 19:617-637

39. Kraemer HC, Yesavage JA, Taylor JL, Kupfer D (2002) How can we learn about developmental processes from cross-sectional studies, or can we? Am J Psychiatry 157:163-171

40. Kramer AF, Larish JF, Strayer DL (1995) Training for attentional control in dual task settings: a comparison of young and old adults. J Exp Psychol Appl 1:50-76
41. Kramer AF, Hahn S, Cohen NJ et al (1999) Aging, fitness, and neurocognitive function. Nature 400:418-419

42. Kramer AF, Colcombe S, McAuley E et al (2003) Enhancing brain and cognitive function of older adults through fitness training. J Mol Neurosci 20:213-221

43. Krampe RT, Rapp MA, Bondar A, Baltes PB (2003) Selektion, Optimierung und Kompensation in Doppelaufgaben. Nervenarzt $74: 211-218$

44. Labouvie EW (1980) Measurement of individual differences in intraindividual changes. Psychol Bull 88:54-59

45. Li KZH, Lindenberger U, Freund AM, Baltes PB (2001) Walking while memorizing: age-related differences in compensatory behavior. Psychol Sci 12:230-237

46. Li KZH, Krampe RT, Bondar A (2005) An ecological approach to studying aging and dual-task performance. In: Engle RW, Sedek G, von Hecker U, McIntosh DN (eds) Cognitive limitations in aging and psychopathology. Cambridge University Press, Cambridge, England, pp 190-218

47. Li S-C, Lindenberger U (1999) Cross-level unification: a computational exploration of the link between deterioration of neurotransmitter systems and dedifferentiation of cognitive abilities in old age. In: Nilsson LG, Markowitsch HJ (eds) Cognitive neuroscience of memory. Hogrefe and Huber, Seattle, pp 103-146

48. Li S-C, Jordanova M, Lindenberger U (1998) From good senses to good sense: a link between tactile information processing and intelligence. Intelligence 26:99-122

49. Li S-C, Lindenberger U, Frensch PA (2000) Unifying cognitive aging: from neuromodulation to representation to cognition. Neurocomputing 32-33:879-890

50. Li S-C, Aggen SH, Nesselroade JR, Baltes PB (2001) Short-term fluctuations in elderly people's sensorimotor functioning predict text and spatial memory performance: the MacArthur successful aging studies. Gerontology 47:100-116

51. Li S-C, Lindenberger U, Hommel B, Aschersleben G, Prinz W, Baltes PB (2004) Transformations in the couplings among intellectual abilities and constituent cognitive processes across the life span. Psychol Sci 15:155-163

52. Li S-C, Lindenberger U, Smith J (2005) Intra-person dynamics across the lifespan. In: Baltes PB, Baumert J, Gigerenzer G, Lindenberger U, Mayr KU (eds) Max Planck Institute for Human Development: research report 2003-2004. Kettler, Bönen, pp 166-174

53. Lindenberger U, Baltes PB (1994) Sensory functioning and intelligence in old age: a strong connection. Psychol Aging 9:339-355

54. Lindenberger U, Pötter U (1998) The complex nature of unique and shared effects in hierarchical regression analyses: implications for developmental psychology. Psychol Methods 3:218-230

55. Lindenberger U, von Oertzen T (2006) Variability in cognitive aging: from taxonomy to theory. In: Craik FIM, Bialstok E (eds) Lifespan cognition: mechanisms of change. University Press, Oxford, UK, pp 297-314

56. Lindenberger U, Marsiske M, Baltes PB (2000) Memorizing while walking: increase in dual-task costs from young adulthood to old age. Psychol Aging 15:417-436

57. Lövdén M, Lindenberger U (2005) Development of intellectual abilities in old age: from age gradients to individuals. In: Wilhelm $\mathrm{O}$, Engle RW (eds) Understanding and measuring intelligence. Sage, Thousand Oaks, CA, pp 203-221

58. Lövdén M, Ghisletta P, Lindenberger U (2004) Cognition in the Berlin Aging Study (BASE): the first 10 years. Neuropsychol Dev Cogn Sect B Aging Neuropsychol Cogn 11:104-133

59. Lövdén M, Schellenbach M, Grossman-Hutter B, Krüger A, Lindenberger U (2005) Environmental topography and postural control demands shape aging-associated decrements in spatial navigation performance. Psychol Aging 20:683-694 
60. Lundin-Olsson L, Nyberg L, Gustafson Y (1997) "Stops walking when talking" as a predictor of falls in elderly people. Lancet 349:617

61. Madden DJ, Blumenthal JA, Allen PA, Emery CF (1989) Improving aerobic capacity in healthy older adults does not necessarily lead to improved cognitive performance. Psychol Aging 4:307-320

62. McAuley E, Kramer AF, Colcombe S (2004) Cardiovascular fitness and neurocognitive function in older adults: a brief review. Brain Behav Immun 18:214-220

63. Mechling H (2005) Körperlich-sportliche Aktivität und erfolgreiches Altern. Bundesgesundheitsblatt Gesundheitsforschung Gesundheitsschutz 48:899-905

64. Molenaar PCM (2004) A manifesto on psychology as idiographic science: bringing the person back into scientific psychology, this time forever. Measurement 2:201-218

65. Molenaar PCM, Huizenga HM, Nesselroade JR (2003) The relationship between the structure of inter-individual and intra-individual variability: a theoretical and empirical vindication of developmental system theory. In: Staudinger UM, Lindenberger $U$ (eds) Understanding human development: dialogues with lifespan psychology. Kluwer, Dordrecht, NL, pp 339-360

66. Moul J, Goldman B, Warren B (1995) Physical activity and cognitive performance in the older population. J Aging Phys Act 3:135-145

67. Neeper SA, Gomez PF, Choi J, Cotman C (1995) Exercise and brain neurotrophins. Nature 373:109

68. Park DC, Polk TA, Mikels JA, Taylor SF, Marshuetz C (2001) Cerebral aging: integration of brain and behavioral models of cognitive function. Dialogues In Clinical Neuroscience 3:151-165
69. Peters A (2002) The effects of normal aging and nerve fibers: a review. J Neurocytol 31:581-593

70. Raz N, Lindenberger U, Rodrigue KM et al (2005) Regional brain changes in aging healthy adults: general trends, individual differences and modifiers. Cereb Cortex 15:1676-1689

71. Reinert G, Baltes PB, Schmidt LR (1996) Critique of a critique of the differentiation hypothesis of intelligence. Z Exp Angew Psychol 13:602

72. Rogers RL, Meyer JS, Mortel KF (1990) After reaching retirement age physical activity sustains cerebral perfusion and cognition. J Am Geriatr Soc 38:123-128

73. Shumway-Cook A, Woollacott M, Kerns KA, Baldwin M (1997) The effects of two types of cognitive tasks on postural stability in older adults with and without a history of falls. J Gerontol A Biol Sci Med Sci 52(4):M232-M240

74. Spirduso WW (1975) Reaction and movement time as a function of age and physical activity level. J Gerontol 43:18-23

75. Spirduso WW, Clifford P (1978) Replication of age and physical activity effects on reaction time and movement time. J Gerontol 33:23-30

76. van Praag H, Kempermann G, Gage FH (1999) Running increases cell proliferation and neurogenesis in the adult mouse dentate gyrus. Nat Neurosci 2:266-270

77. von Oertzen T, Lindenberger U (2005) On detecting causes of developmental change from cross-sectional regression models. 58th Annual Scientific Meeting of The Gerontological Society of America, Orlando, Florida

78. Welford AT (1958) Ageing and human skill. Oxford University Press, London

79. Wickens CD (1991) Processing resources and attention. In: Damos D (ed) Multiple-task performance. Taylor \& Francis, London, pp 3-34 